\title{
Actualización acerca de colistina (polimixina E): aspectos clínicos, PK/PD y equivalencias
}

\author{
Dres. Julio Medina*, Daniela Paciel†, Ofelia Noceti, Gloria Rieppi§
}

\section{Resumen}

En los últimos diez años, en esta era posantibiótica, la colistina ha resurgido como un último recurso frente a infecciones por patógenos como Pseudomonas aeruginosa, Acinetobacter baumannii complex y enterobacterias productoras de carbapenemasas. Cayó en desuso previamente dados sus efectos adversos potencialmente graves, como la nefro y neurotoxicidad, pero hoy revive como parte fundamental de los planes antibióticos frente a patógenos extremadamente resistentes. El aumento de su uso conlleva a la emergencia de resistencia, encontrándonos frente a una situación potencialmente catastrófica, en particular dada la recientemente descrita presencia de plásmidos transferibles entre especies conteniendo genes que confieren resistencia a colistina (gen $m c r-1$ ), mecanismo detectado también en nuestro país.

En la presente revisión, basados en los conocimientos actuales sobre la farmacocinética y la farmacodinamia, se describe en detalle la dosificación apropiada con necesidad de realizar dosis carga para alcanzar los niveles terapéuticos adecuados, así como aspectos prácticos de la administración en casos de meningitis/ventriculitis posquirúrgica y del empleo por vía nebulizada para el tratamiento de la neumonía asociada a la ventilación. Se destaca la necesidad de su uso combinado con otro antibiótico activo in vitro, en particular en pacientes críticos y en aquellos con un clearance de creatininemia mayor a 80 $\mathrm{ml} / \mathrm{min}$. La biterapia es necesaria en particular si la concentración inhibitoria mínima (CIM) del patógeno es mayor a $1 \mathrm{mg} / \mathrm{l}$, debido al riesgo de subdosificación y emergencia de resistencia intratratamiento.

En una segunda sección se aborda la complejidad de la dosificación en función de las distintas presentaciones comercializadas a nivel nacional que han conducido a errores en la posología, con un riesgo mayor de eventual toxicidad. Con el objetivo de mejorar la comprensión referente a la rotulación de la dosis a administrar de colistina se revisaron los insertos y envases primarios de las presentaciones de colistina comercialmente disponibles en Uruguay, a efectos de solicitar formal y documentalmente a las empresas farmacéuticas representantes, se expidan en cuanto a los contenidos de colistina (droga activa) y de su profármaco, el colistimetato sódico (CMS). Finalmente se elaboraron una serie de recomendaciones en cuanto a la información que a entender de los autores debieran exhibir las distintas presentaciones de colistina comercialmente disponibles para no inducir a errores en la prescripción.

Palabras clave: COLISTINA

MONITOREO DE DROGAS

PARÁMETROS PK/PD

FARMACORRESISTENCIA BACTERIANA MÚLTIPLE
Key words: $\quad$ COLISTIN

DRUG MONITORING

PK/PD PARAMETERS

MULTIPLE BACTERIAL DRUG RESISTANCE

\footnotetext{
* Prof. Director de la Cátedra de Enfermedades Infecciosas. Facultad de Medicina, Universidad de la República, Uruguay.

† Prof. Adj. de la Cátedra de Enfermedades Infecciosas. Facultad de Medicina, Universidad de la República, Uruguay.

‡ Farmacóloga. Clínica Servicio de Enfermedades Hepáticas, Centro Nacional de Trasplante Hepático, Hospital Central de las Fuerzas Armadas,

Uruguay.

$\S$ Prof. Agda. de la Cátedra de Medicina Intensiva, Facultad de Medicina, Universidad de la República. Uruguay.

Los autores declaran no tener conflictos de interés.

Correo electrónico : jcmedina@fmed.edu.uy

Recibido: 8/5/17

Aceptado: 12/6/17
} 


\section{Introducción}

En los últimos años la emergencia de resistencia a carbapenemes en patógenos como Klebsiella pneumoniae, Acinetobacter baumanii o Pseudomonas aeruginosa ha llevado a reutilizar antimicrobianos en desuso. En particular para el tratamiento de enterobacterias productoras de carbapenemasas tipo KPC o NDM (New Delhi metalobetalactamasa), así como de bacilos gramnegativos no fermentadores, los clínicos han tenido que emplear antimicrobianos como la fosfomicina, la tigeciclina y las polimixinas, ya sea en terapia combinada doble o triple, dada la escasez de moléculas farmacológicamente activas disponibles ${ }^{(1)}$.

En el año 2009 se aísla por primera vez en nuestro país Klebsiella pneumoniae, productora de carbapenemasa tipo KPC (Kpn, KPC), con reportes de brotes aislados en distintas instituciones desde ese momento. Si bien no hay evidencias de endemicidad, como en otros países de la región, Uruguay no está exento de una diseminación más extensa en los años próximos. Asimismo, aunque en brotes aislados, también se han detectado otros tipos de carbapenemasas tanto en enterobacterias (metalocarbapenemasas tipo NDM), así como en Pseudomonas aeruginosa y Acinetobacter baumannii (metalobetalactamasas tipo VIM) ${ }^{(2)}$.

Con la premisa de administrar un tratamiento eficaz y disminuir la aparición potencial de resistencia bajo tratamiento y minimizar los potenciales efectos adversos, es que en el año 2011 se elabora el Consenso Uruguayo para el Uso de Antimicrobianos en Infecciones por Microorganismos Multi y Panresistentes y las Guías para el Tratamiento de Bacterias productoras de $\mathrm{KPC}^{(3)}$. Posteriormente, en el año 2013 se publica un consenso intersociedades científicas en el que participa Uruguay, enfocado en el tratamiento de enterobacterias productoras de carbapenemasas ${ }^{(4)}$. En ambos documentos se plasma el problema referente a la escasez de alternativas antibióticas eficaces, lo que conlleva a la construcción de planes alternativos. De los fármacos que integran el backbone de estas estrategias, se destacan la polimixina B y E o colistina. Respecto a estas últimas, se prefiere el uso de la polimixina $\mathrm{B}$ dado su mejor perfil farmacocinético $(\mathrm{PK}) /$ farmadodinámico $(\mathrm{PD})$, sin necesidad de administrar dosis carga ni ajuste de dosis en caso de injuria renal y por su menor riesgo de nefrotoxicidad ${ }^{(5,6)}$.

La colistina (polimixina E) es la única disponible actualmente en nuestro país. Su uso en franco aumento ha permitido profundizar en el conocimiento de su perfil farmacocinético (PK), farmacodinámico (PD) y toxicodinámico en vistas optimizar su uso clínico, dado que la administración de dosis que conducen a concentraciones subterapéuticas en los sitios de acción contribuyen a la selección de cepas resistentes o a sobreinfección, mientras que las que resultan en sobredosificación pueden producir toxicidad, particularmente neuro y nefrotoxicidad $^{(7,8)}$. El aumento significativo en su uso también ha puesto de manifiesto la emergencia de resistencia, cuyo mecanismo es principalmente cromosómico, aunque en los últimos dos años se ha descrito resistencia a colistina codificada en plásmidos, es decir en elementos móviles transferibles aun entre diferentes especies, lo que puede reducir todavía más el arsenal terapéutico actualmente disponible ${ }^{(9)}$. Este mecanismo ya se ha detectado en muestras clínicas en Uruguay y en la región ${ }^{(10)}$.

En cuanto a la comercialización de la colistina, luego de su reintroducción en el mercado, se ha generado confusión en relación con los diferentes nombres del fármaco y su concentración en las diferentes presentaciones comerciales, así como en la información contenida en los insertos y etiquetas, y por consecuencia a la dosis recomendada a administrar debido a las diferentes unidades de medida ${ }^{(7)}$. Los trabajos publicados a la fecha de este informe y de las presentaciones comercialmente disponibles de colistina en Uruguay reflejan también esta confusión dado que expresan la concentración del fármaco en diferentes unidades, como miligramos o unidades internacionales, no explicitándose claramente si se refiere al contenido de los viales en términos de colistina base (droga activa) o de su profármaco, el colistimetato sódico $(\mathrm{CMS})^{(11,12)}$.

Esta heterogeneidad puede inducir a errores comprometiendo el perfil de eficacia y la seguridad del paciente a tratar. El objetivo de esta revisión es presentar en forma clara los nuevos aspectos PK/PD de este fármaco y esclarecer las equivalencias de las presentaciones comercializadasa nivel nacional.

\section{Características de la colistina}

\section{Mecanismo de acción}

La colistina es un polipéptido cíclico perteneciente al grupo de las polimixinas, que por sus propiedades tensoactivas tiene la capacidad de alterar la permeabilidad de la pared de las bacterias gramnegativas sensibles, que presentan una capa externa conformada por lipopolisacáridos (LPS). Las polimixinas conducen a la muerte celular de la bacteria por la atracción electrostática ejercida entre la carga positiva de los grupos amino y los aniones fosfato y carboxilato que componen la capa de lipopolisacáridos. En definitiva, la interacción entre los ácidos grasos y otras regiones hidrofóbicas de la colistina con los dominios hidrofóbicos de la capa de LPS provoca modificaciones en la estructura de la pared celular, que promueven la pérdida de su contenido, el ac- 
ceso del fármaco y en consecuencia su efecto bacterici$\mathrm{da}^{(13,14)}$.

\section{Espectro antibacteriano}

Si bien el espectro antimicrobiano comprende bacterias gramnegativas aerobias, como las enterobacterias, y bacilos gramnegativos no fermentadores, como Acinetobacter baumannii y Pseudomonas aeruginosa, debe tenerse en cuanta que hay patógenos naturalmente resistentes a colistina dentro de estos grupos, como Stenotrophomonas maltophilia, Proteus sp, Providencia sp, Serratia sp y Burkholderia $\mathrm{sp}^{(15)}$. Quedan fuera de su cobertura también los cocos grampositivos y los anaerobios.

Las polimixinas se consideran fármacos de último recurso para el tratamiento de pacientes con infecciones causadas por bacterias gramnegativas multirresistentes. En función de ello, en el año 2016 se estableció un grupo de trabajo conjunto entre el Clinical and Laboratory Standards Institute (CLSI) y el European Committee on Antimicrobial Susceptibility Testing (EUCAST) que abordó los retos globales asociados a la colistina, como la identificación de métodos de testeo fiables así como el establecimiento de puntos de corte de la $\mathrm{CIM}^{(16)}$. Se determinó como punto de corte de la CIM para considerar la cepa sensible en $\leq 2 \mathrm{mg} / \mathrm{l}$ tanto para enterobacterias como para Pseudomonas spp. y Acinetobacter baumannii complex y como resistente con una CIM > $2 \mathrm{mg} / \mathrm{l}^{(17,18)}$. Es importante destacar que el uso de colistina debería reservarse para el tratamiento específico de infecciones producidas por microorganismos resistentes a los betalactámicos, en particular los carbapenemes, o como parte del tratamiento empírico de pacientes con infecciones graves (siempre asociada a otros antimicrobianos) si se sospecha la participación de un microorganismo con ese perfil de resistencia ${ }^{(4-19)}$.

\section{Mecanismos de resistencia}

La resistencia adquirida a colistina se ha mantenido en bajos niveles a lo largo de los años a pesar del aumento significativo en su uso. Hasta hace poco se sabía que la resistencia a las polimixinas, en general, se daba por mutaciones a nivel cromosómico, no habiéndose informado acerca de la transferencia horizontal de genes que otorgaran resistencia a las mismas. La resistencia a colistina se explica por mutaciones en los genes que conducen a modificaciones en la capa de LPS, sitio de acción de esta molécula ${ }^{(13,20)}$.

Adicionalmente a esta modalidad, en noviembre de 2015 se informó sobre la aparición de un nuevo mecanismo de resistencia a colistina a través de plásmidos, relacionado al gen mcr-1 (Mobile Colistin Resistance), productor de una enzima responsable de la resistencia, por lo que se puede diseminar fácilmente la misma a otras bacterias ${ }^{(9-22)}$. Desde abril de 2016 en nuestro continente se ha identificado este mecanismo de resistencia en $E$. coli y otras enterobacterias, aisladas a partir de muestras de alimentos y animales, pero también de muestras clínicas de pacientes. En nuestro país se ha detectado este tipo de mecanismo de resistencia en muestras clínicas humanas ${ }^{(10)}$. Hasta el momento no se han reportado brotes o muertes causadas por microorganismos portadores de este gen, si bien el riesgo mayor que se enfrenta es la diseminación de este gen mediante plásmidos a otras cepas más virulentas o incluso a clones hiperepidémicos ${ }^{(21)}$. Si llegara a darse esta situación, el uso de colistina como estrategia farmacológica quedaría obsoleta para el tratamiento de patógenos extremadamente resistentes con las implicancias de morbimortalidad que esto conlleva.

La heterorresistencia es un fenómeno a destacar en el uso de la colistina. Este término se emplea para referirse a las poblaciones bacterianas que contienen una mayoría de bacterias inhibidas en concentraciones por debajo del punto de corte de sensibilidad, junto con otro pequeño número de microorganismos (en torno a 1 por cada $10^{3}-10^{7}$ ) que son resistentes ${ }^{(23)}$. Sin embargo, no se cuenta con un método estandarizado para su detección. En este contex to el antimicrobiano actuaría como agente de selección, eliminando la población más sensible y permitiendo la supervivencia de la fracción mutante, lo que podría deberse también a un fenómeno de persistencia. La exposición a concentraciones del fármaco insuficientes podría representar un factor de riesgo para el enriquecimiento in vivo de la población resistente, y esto podría relacionarse con fracaso terapéutico ${ }^{(20)}$. Los aislamientos de $A$. baumannii de pacientes tratados previamente con colistina presentan un mayor grado de heterorresistencia ${ }^{(24)}$. Esta es en parte la base teórica para el uso en terapia combinada de colistina, en particular en infecciones graves.

\section{Farmacocinética y farmacodinamia}

La colistina es un fármaco de estrecho margen terapéutico, por lo que se requiere mantener un balance entre las concentraciones necesarias en el sitio de acción para lograr la eficacia terapéutica minimizando la emergencia de resistencia y al mismo tiempo evitar su toxicidad.

La colistina se administra en viales conteniendo colistimetato sódico (nombres alternativos: sulfometato de colistina, colismetansulfonato pentasódico, metansulfonato de colistina). El colistimetato sódico (CMS) es un profármaco inactivo que se transforma en medio acuoso (in vitro e in vivo) a colistina, que es la molécula que ejerce el efecto farmacológico ${ }^{(25)}$.

El CMS se elimina a nivel renal por filtración glomerular y por secreción a nivel tubular. En individuos con 
función renal normal el clearance renal de CMS es mucho más rápido que su conversión a colistina, recuperándose alrededor de un $80 \%$ a las 24 horas de forma prácticamente incambiada, lo que se debe tener en cuenta si el sitio de infección es urinario. Por el contrario, la eliminación renal de colistina es prácticamente inexistente ya que tiene muy extensa reabsorción tubular y un clearance predominantemente extrarrenal, por un mecanismo no bien conocido aún ${ }^{(14)}$. Esta característica debería considerarse en la posología de pacientes con disfunción renal, ya que hay menos filtración de CMS y, por lo tanto, menor reabsorción renal de colistina. Es difícil conocer el impacto que la disminución de la función renal (y por ende el aumento de CMS) tiene en la cantidad de colistina formada sobre la cual la eliminación renal contribuye poco en su clearance $^{(7,12)}$. El CMS y la colistina sódica se eliminan por medio de la diálisis, por lo tanto, cuando se aplican terapias de reemplazo renal se deberían administrar dosis adicionales a la dosis diaria administrada ajustada al clearance de creatinina.

Por otra parte, la colistina administrada por vía nebulizada o intratecal tiene una mínima absorción sistémica. En particular la administración para nebulizar potenciaría el efecto local, minimizando efectos adversos renales $^{(14)}$.

Como la mayoría de las infecciones se desarrollan a nivel del fluido intersticial, las concentraciones plasmáticas subestiman los valores reales en los sitios de acción. Esta situación se agrava en pacientes con alteración de la microcirculación (shock séptico), donde se dificulta la penetración tisular de la colistina. En la sepsis, además, se pueden agregar factores que determinan una disminución de la concentración sérica de colistina por aumento del volumen de distribución (hiperpermeabilidad capilar, tercer espacio, drenajes, abdomen abierto, etc.) y mayor eliminación renal por aumento del gasto cardíaco (hiperdinamia, administración de vasopresores/fluidos). Esta situación particular puede conducir, entre otros factores, a una falla del tratamiento independientemente de la sensibilidad del microorganismo al antibiótico.

A nivel neurológico, el pasaje de colistina a través de la barrera hematoencefálica es pobre debido a las características de la molécula. Se han reportado concentraciones de 5\% de las plasmáticas en el líquido cefalorraquídeo (LCR) en casos de meninges con inflamación discreta, y de hasta $25 \%$ si la inflamación es intensa ${ }^{(26,27)}$.Es decir, que la resolución de la meningitis con tratamiento exclusivamente intravenoso sería posible en caso de $A$. baumannii con $\mathrm{CIM}<0,5 \mathrm{mg} / \mathrm{L}^{(26)}$. La administración intratecal (a través de drenaje lumbar externo o intraventricular en pacientes con drenaje ventricular externo) representa una alternativa terapéutica de la meningi- tis/ventriculitis por A. baumannii con CIM mayor u otro patógeno solo sensible a colistina, empleando dosis bajas ya que el volumen de este espacio y la velocidad de recambio del LCR son pequeños ${ }^{(28-30)}$.

\section{Relación farmacocinética/farmacodinamia}

La acción bactericida de colistina es concentración-dependiente. A partir de modelos de infección in vitro se ha reportado que el mejor parámetro PK de colistina es la relación de concentración en el área bajo la curva en función del tiempo (AUC) sobre la CIM (fAUC/CIM).

Para microorganismos con CIM de $1 \mathrm{mg} / 1$ se requeriría un AUC 0-24h/CMI = $60 \mathrm{mg} \times \mathrm{h} / \mathrm{l}$. Para ello, es necesario alcanzar una concentración plasmática media mínima en estado estacionario de $2,5 \mathrm{mg} / 1$, aunque en algunos casos podría ser necesario alcanzar $4 \mathrm{mg} / \mathrm{l}$ de concentraciones plasmáticas del fármaco total, es decir unido a proteínas plasmáticas y libre (biodisponible) ${ }^{(26)}$, lo que evidencia un estrecho rango por encima de la CIM.

Un objetivo plasmático de colistina de $2,5 \mathrm{mg} / \mathrm{l} \mathrm{se}$ traduce in vitro a una actividad bacteriostática contra $\mathrm{BGN}$ con CIM entre $0,5-2,0 \mathrm{mg} / \mathrm{l}^{(31)}$. Como no se dispone de estudios que evalúen la eficacia y seguridad a dosis mayores, este hecho es un argumento más para que se recomiende su uso en terapias combinadas ${ }^{(32,33)}$.

En voluntarios sanos por administración endovenosa, la concentración plasmática máxima (Cmáx) se alcanza en el entorno de dos horas, mientras que en pacientes críticos se observa un retraso de hasta siete ho$\operatorname{ras}^{(13)}$. Debido a la lenta conversión de CMS a colistina, la larga semivida de eliminación de colistina y el tiempo para alcanzar el estado estacionario plasmático hace necesaria la administración de una dosis carga para compensar estos efectos durante los primeros días de tratamiento y minimizar en alcanzar concentraciones terapéuticamente óptimas ${ }^{(14)}$. El clearance de creatinina es la variable clave para calcular la dosis de mantenimiento, sin embargo la dosis de carga depende del peso.

\section{Reacciones adversas}

Nefrotoxicidad (> 10\%). Como la eliminación del CMS es renal, existe riesgo potencial de nefrotoxicidad, que es mayor cuando las concentraciones plasmáticas de colistina son superiores a 2,5-3 mg/l y en pacientes con un clearance de creatinina menor a $80 \mathrm{ml} / \mathrm{min}^{(5,14)}$. También se ha asociado a episodios de hematuria, proteinuria, oliguria y falla renal aguda debidos a necrosis tubular aguda, que generalmente revierten al adaptar la posología. En la gran mayoría de los casos la falla renal aparece en los primeros siete días de tratamiento y la función renal se recupera tras la suspensión del fármaco en al menos $90 \%$ de los casos ${ }^{(34)}$. 
Neurotoxicidad (10\%). Cuando las concentraciones plasmáticas superan el intervalo de seguridad, los pacientes bajo tratamiento con colistina pueden desarrollar vértigo, parestesia facial transitoria, alteraciones del lenguaje, inestabilidad vasomotora, alteraciones visuales, confusión, psicosis, apnea y convulsiones, entre $\operatorname{otros}^{(34,35)}$. En particular, con la administración por vía intraventricular o intratecal existe un riesgo de $10 \%$ de desarrollo de meningitis química, con presencia de fiebre, estado mental alterado, aumento del conteo de leucocitos y disminución de los niveles de glucosa en el $\mathrm{LCR}^{(28)}$.

Respiratorio (10\%). La administración de colistina para nebulización puede provocar diferentes grados de hiperreactividad, tal como tos seca o broncoconstricción.

Hipersensibilidad (2\%). Se han constatado casos de erupciones exantemáticas, rush, prurito, urticaria o fiebre.

\section{Interacciones, embarazo / lactancia, contraindicaciones, sobredosificación(36)}

Como la colistina favorece la liberación de acetilcolina, debe contemplarse el aumento potencial de bloqueo neuromuscular, que puede conducir a parálisis respiratoria durante el uso concomitante con bloqueantes neuromusculares. La coadministración con otros fármacos, como aminoglucósidos, anfotericina B y furosemide, puede potenciar los efectos neuro y nefrotóxicos.

Asimismo, la colistina atraviesa la placenta y se detecta a nivel de la leche materna.

Las principales contraindicaciones son la miastenia gravis, debido a que el CMS reduce la liberación presináptica de acetilcolina a nivel de la unión neuromuscular y la hipersensibilidad conocida a colistina.

El tratamiento por sobredosificación tiene como fin la eliminación de colistina mediante diuresis osmótica, diálisis peritoneal o hemodiálisis ${ }^{(31)}$.

\section{Monitoreo terapéutico}

La monitorización de la concentración plasmática sería una herramienta imprescindible para el clínico debido al estrecho margen terapéutico del fármaco. En Uruguay no se ha implementado la medida de colistina en sangre. Al momento solo se cuenta con datos de concentración sérica de colistina aportados por estudios de investigación $^{(37,38)}$. Por ello, es imprescindible realizar un estrecho monitoreo clínico de uno de sus principales efectos adversos, la disfunción renal. Un aumento de la creatininemia $\geq 0,3 \mathrm{mg} / \mathrm{dL}$ (en un período de 48 horas) $\mathrm{o} \geq 50 \%$ del valor basal (en un período $\leq 7$ días) obliga al ajuste de dosis de colistina de acuerdo a la eliminación renal. La fórmula para el cálculo de clearance de creatinina se puede realizar con cuatro a ocho horas de diuresis, expresado en $\mathrm{ml} / \mathrm{min}$ y como concentración de creatinina en orina por volumen urinario en $\mathrm{ml}$ del período estudiado/concentración de creatinina plasmática por tiempo en minutos.

\section{Posología y vías de administración}

Las dosis a administrar y la duración del tratamiento se deben adaptar a la situación clínica dada por la gravedad, a factores vinculados al paciente tales como edad, peso y función renal, a la penetración en el sitio de infección y a la respuesta clínica.

Actualmente existen tres diferentes recomendaciones a nivel internacional de cómo calcular la dosis de colistina por el Grupo de Estudio PK, por la Food and Drugs Administration (FDA) y la European Medicine Agency (EMA) ${ }^{(26-39)}$. Las tres tienen como base el clearance de creatinina con diferentes subdivisiones, mientras que la FDA toma en cuenta además el peso del paciente $^{(26,39)}$. El peso a tener en cuenta es el peso ideal, es decir el peso adecuado al sexo y a la altura del paciente. En nuestro país se ha generalizado el cálculo en base a miligramos de colistina en función del peso, es decir de conformidad a las recomendaciones dadas por la FDA.

\section{Vía intravenosa}

Un régimen posible a recomendar, basado en $\mathrm{mg}$ de colistina o colistina base activa (CBA), es el de 2,5 a 5 $\mathrm{mg} / \mathrm{kg} /$ día fraccionada idealmente en dos dosis de acuerdo a la gravedad de la infección ${ }^{(1,40)}$. En el caso de pacientes con infección grave es complicado recomendar la posología más adecuada. La compleja farmacocinética del CMS y de la colistina, ya comentada, sumado a factores vinculados al sitio de infección, el frecuente aumento del volumen de distribución del fármaco, la alteración de la microcirculación en el shock séptico y el cambiante clearance de creatinina en un mismo paciente $\mathrm{y}$ en algunos casos la necesidad de terapia de reemplazo renal (TRR) dificultan la recomendación de una posología exenta de riesgos (dosis subterapéutica vs dosis con severos efectos adversos). Lo que es claro es que se debería asociar otro antimicrobiano en particular cuando la CIM a colistina para la bacteria a tratar es $\geq 1$ $\mathrm{mg} / \mathrm{L}$ o el clearance de creatinina es $\geq 80 \mathrm{ml} / \mathrm{min}$, dado que se ha observado que con dosis de colistina recomendadas por FDA y EMA de $5 \mathrm{mg} / \mathrm{kg}$ y $300 \mathrm{mg} /$ día, respectivamente, las concentraciones plasmáticas de colistina $\geq 1 \mathrm{mg} / \mathrm{l}$ no se alcanzan en el $80 \%$ de los ca$\operatorname{sos}^{(26)}$. Por otra parte, se recomiendan también dosis máximas día que no deberían superar los 360 mg, según el Grupo de Estudio PK ${ }^{(39)}$.

Dosis carga: como los niveles terapéuticos se alcanzan transcurridos dos a tres días del inicio de la adminis- 
Tabla 1. Dosificación de colistina según función renal, referidas en mg de colistina o colistina base activa.*

\begin{tabular}{|c|c|c|c|}
\hline Clearance de creatinina (I/min) & $\begin{array}{l}\text { Grupo de Estudio PK (dosis diaria } \\
\text { máxima dividida en dos dosis) }\end{array}$ & EMA (dosis dividida en dos) & FDA (según peso ideal) \\
\hline$\geq 90$ & 360 mg/día & & 2,5-5 mg/kg/día dividido en dos dosis \\
\hline 80 a $<90$ & 340 mg/día & 300 mg/día & \\
\hline $70 a<80$ & 300 mg/día & & 2,5-3,8 mg/kg/día dividido en dos dosis \\
\hline $60 \mathrm{a}<70$ & 275 mg/día & & \\
\hline $50 a<60$ & 245 mg/día & & \\
\hline $40 a<50$ & 220 mg/día & 183-250 mg/día & $2,5 \mathrm{mg} / \mathrm{kg}$ dividido en dos dosis \\
\hline $30 a<40$ & 195 mg/día & & \\
\hline $20 a<30$ & 175 mg/día & 150-183 mg/día & $1,5 \mathrm{mg} / \mathrm{kg}$ cada 36 horas \\
\hline $10 a<20$ & 160 mg/día & & \\
\hline $5 a<10$ & 145 mg/día & 117 mg/día & No recomiendan su uso \\
\hline$<5$ & 130 mg/día & & \\
\hline
\end{tabular}

* Adaptado de Guilbert DN, Chambers HF, Eliopoulos GM, Saag MS, Pavia AT. The Sanford Guide to Antimicrobial Therapy. 2017. 47th Edition. ISBN-13: 978-1944272012

tración del fármaco, resulta imprescindible administrar una dosis carga. La dosis carga de colistina calculada como colistina base activa (CBA) debe realizarse siempre independientemente de la función renal a $5 \mathrm{mg}$ de colistina por $\mathrm{kg}$ de peso ideal.

Dosis de mantenimiento: la dosis diaria de mantenimiento se debe iniciar a las 12 horas de administrada la dosis carga, 2,5 a $5 \mathrm{mg} / \mathrm{kg}$ dividida en dos dosis/día. En los pacientes críticos o en infecciones graves sugerimos utilizar el rango superior de la dosis calculada. Usualmente las dosis de mantenimiento serán de 300 a 400 mg de colistina/día según situación clínica y datos microbiológicos. La dosis se debe reconstituir inmediatamente antes de su administración y diluir en suero salino a $0,9 \%$ (aunque es posible emplear ringer lactato o dextrosa a $5 \%$ ).

Para el ajuste de dosis de mantenimiento en caso de insuficiencia renal se sugiere aplicar lo expresado en la tabla 1, donde se resumen las recomendaciones del Grupo de Estudio PK, la FDA y la EMA ${ }^{(26,39)}$.

En caso de hemodiálisis intermitente (HDI) la dosis adicional poshemodiálisis debe ser equivalente a un $30 \%$ de la dosis diaria si se administró durante la última hora de la HDI o 50\% de la dosis día si la dosis adicional se administró una vez finalizada la $\mathrm{HDI}^{(31)}$.

\section{Vía intratecal (intraventricular o intrarraquídea)}

Para el tratamiento de meningitis/ventriculitis cuando el paciente tiene un drenaje ventricular o intrarraquídeo se puede administrar por vía intratecal asociado o no a la vía intravenosa. La dosis consensuada es de $10 \mathrm{mg} /$ día, aunque hay reportes del uso del doble de esta do$\operatorname{sis}^{(28-30)}$. Considerando la posibilidad de cepas formadoras de biofilms, estos catéteres deben ser retirados lo más precozmente posible. La mayoría de los protocolos de tratamiento para meningitis posneuroquirúrgica a bacterias gramnegativas solo sensibles a colistina asocian al tratamiento intravenoso cuando es posible el tratamiento tópico intratecal.

La Sociedad Americana de Enfermedades Infecciosas recomienda para el tratamiento de meningitis bacteriana dosis de $10 \mathrm{mg}$ diarios de colistina administrados por vía intraventricular, durante 21 días, con ajustes acorde a la respuesta clínica del paciente y hasta la obtención de tres cultivos de LCR negativos extraídos en días separados ${ }^{(41)}$. Esta estrategia ha mejorado las tasas de mortalidad y curación ${ }^{(35)}$.La Cátedra de Medicina Intensiva de la Universidad de la República recomienda la administración de $15 \mathrm{mg} /$ día en una dosis preparada en solución salina a $0,9 \%$ en un volumen final de $2 \mathrm{ml}$; previo a esta administración se debe drenar un volumen de LCR de unos $5 \mathrm{ml} \mathrm{y}$, una vez finalizada la administración de colistina, administrar $2 \mathrm{ml}$ de suero salino a $0,9 \%$ para evitar que la droga quede en el catéter. El sistema debe permanecer cerrado durante una a tres horas (de acuerdo a tolerancia clínica por la presión intracraneana). No debe emplearse la misma dilución de una administración a otra, siendo necesario descartarla ${ }^{(42)}$. 


\section{Vía inhalada (nebulización)}

Para nebulizar se la emplea para el tratamiento de las infecciones respiratorias, tanto en la traqueobronquitis asociada al ventilador (TAV) como para la neumonía asociada al ventilador (NAV). La coadministración por vía endovenosa se apoya en los resultados de los metaanálisis de las guías 2016 para el tratamiento de la neumonía asociada al respirador ${ }^{(43)}$. No haremos referencia a la indicación en fibrosis quística.

La eficacia y seguridad de esta modalidad de administración es el resultado de la asociación de un fármaco, su formulación y el dispositivo para su aplicación; sin embargo, es altamente dependiente de factores relacionados con el paciente ${ }^{(44)}$. Los sistemas de nebulización descritos para dispensar la formulación son del tipo neblina suave: jet, malla y ultrasónico, los cuales cuentan con diferentes mecanismos de funcionamiento, traduciéndose en las características de su performance ${ }^{(45)}$. Las presentaciones de $50 \mathrm{mg}, 1$ y 2 MUI se reconstituyen en $2 \mathrm{ml}$ de agua para inyección (reconstituyente acoplado a la presentación). Una vez obtenida la solución reconstituida debe emplearse inmediatamente, ya que la formulación solamente admite conservación entre $2{ }^{\circ} \mathrm{C}$ y $8{ }^{\circ} \mathrm{C}$ (heladera) durante 24 horas, debiendo descartarse al término de este período a efectos de no comprometer su estabilidad. Para su administración, introducir la solución en la cámara de un nebulizador homologado con una fuente con suministro de aire u oxígeno. Descartar el remanente no inhalado, si corresponde. La dosis a aplicar es de $50 \mathrm{mg}$ ( 1 a 2 MUI) cada 8 o 12 horas. Se sugiere agregar un broncodilatador en forma previa para facilitar la penetración. Tener en cuenta que la farmacocinética de la formulación puede variar dependiendo del sistema de nebulización empleado, el volumen de llenado y la concentración de la solución.

\section{Tratamiento de la neumonía asociada a la ventilación}

Existen reportes de estudios que demuestran la cura clínica de la NAV administrando colistina por vía intravenosa ${ }^{(46)}$. Sin embargo, es sabido, a partir de medidas de colistina endovenosa en fluidos epiteliales, que alcanza poca penetración pulmonar; a este respecto se ha advertido sobre la interpretación de estos resultados debido a que la concentración de colistina obtenida del lavado bronquiolo-alveolar (LBA) está diluida y, por lo tanto, se encuentra en el límite de cuantificación del ensayo. Como la colistina se une a los tejidos (incluido el pulmón) es posible que el fármaco unido sea mucho mayor que la colistina libre $^{(14)}$. Por lo expuesto, para tratar una NAV por un microorganismo multirresistente, se debe administrar colistina por vía inhalatoria e intravenosa.
En estas condiciones el fármaco reconstituido se administra a través del tubo de inspiración en pacientes ventilados; aquí el seteo del equipo es crucial para lograr la eficacia del tratamiento, donde el volumen del ventilador (usualmente $>500 \mathrm{ml}$ ) debe asegurar la entrega del fármaco a nivel pulmonar y la relación inspiración-espiración debe regularse a efectos de aumentar el tiempo de inspiración ${ }^{(46)}$. En caso de síndrome por distrés respiratorio, el volumen deberá mantenerse a $6 \mathrm{ml} / \mathrm{kg}$ y la velocidad de flujo inspiratoria por debajo de $80 \mathrm{l} / \mathrm{min}$, siendo la óptima $40 \mathrm{l} / \mathrm{min}$, sincronizando la nebulización con el flujo inspiratorio. Se recomienda que el nebulizador se coloque a $10-15 \mathrm{~cm}$ del tubo en $\mathrm{Y}$ en la rama inspiratoria en caso de nebulización continua. La evidencia indica que la eficacia de aerosolización del fármaco mejora si se apaga el humidificador durante la nebulización, es decir que para que la administración sea efectiva en el paciente ventilado, se deben cumplir condiciones especiales ${ }^{(47,48)}$. Muchas veces estos ajustes óptimos no son tolerados por los pacientes (como aquellos con hipoxemia severa o distrés) y requieren sedación profunda y a veces curarización, lo que puede prolongar la duración de la ventilación mecánica ${ }^{(48)}$. Si no se coloca un filtro en la rama espiratoria, puede provocar obstrucción y potencial fallo del respirador, con consecuencias eventualmente fatales para el paciente.

Dentro de los nebulizadores disponibles se cuenta con tres tipos, los cuales proveen diferente tamaño de gota $(\mu)$, cantidad de producto dispensado (mUI), porcentaje de micropartículas $(<0,5 \mu)$, tiempo y tasa de distribución (mUI/minuto) ${ }^{(45)}$. La eficacia de aerosolización, limpieza del nebulizador, velocidad de flujo, sistema de compresión, volumen residual y postura del paciente también influyen en la calidad-eficacia de la administración ${ }^{(46-48)}$. En particular, si se utilizan los del tipo malla vibratoria y ultrasonido se debe tener en cuenta el calentamiento de la solución reconstituida con afectación de su estabilidad. Los equipos de malla vibratoria tipo Respironics I neb Adaptive Aerosol Delivery deberían emplearse con cámara de inhalación de 0,3 o $0,5 \mathrm{ml}$ dependiendo del modelo. De todas formas debe considerarse que aún la evidencia que sustenta el uso de la terapia inhalada para el tratamiento de NAV es escasa y han surgido recientemente revisiones en contra de la $\operatorname{misma}^{(49)}$.

\section{Problemas surgidos de la rotulación del fármaco comercializado}

Las dosis recomendadas de colistina están expresadas en términos de actividad de colistina base (CBA) y el profesional debería realizar los ajustes según las características del producto disponible en cada institución. Por lo tanto, resulta difícil recomendar la posología en 
Tabla 2. Equivalencia entre CMS y actividad de colistina base.

\begin{tabular}{ccc}
\hline Actividad de colistina base (CBA) & $\begin{array}{c}\text { Colismetato sódico } \\
\text { (CMS) (mg) }\end{array}$ \\
\cline { 1 - 2 }$(\mathrm{mg})$ & $(\mathrm{UI})$ & 1 \\
\cline { 1 - 3 } 0,4 & $\sim 12.500$ & 12 \\
5 & $\sim 150.000$ & 80 \\
34 & $\sim 1.000 .000$ & 240 \\
100 & $\sim 3.000 .000$ & 360 \\
150 & $\sim 4.500 .000$ & 720 \\
300 & $\sim 9.000 .000$ & \\
\hline
\end{tabular}

mg de colistina, ya que la mayoría de los estudios que miden colistina en sangre emplean colistina en mUI de potencia. En Europa la presentación de colistina se expresa exclusivamente en millones de unidades internacionales de CMS, a diferencia de lo que sucede en las Américas, Australia y en el Sudeste Asiático, que se expresa en miligramos de actividad de colistina base.

A título de ejemplo, en Argentina, la Sociedad Argentina de Infectología (SADI), habiendo detectado algunas inconsistencias en las presentaciones comerciales de colistina para uso parenteral (diferencias y controversias en la información de prospectos, expresión de la fórmula cualicuantitativa, posología, ajuste de dosis en insuficiencia renal), emitió un alerta a este respecto, decidiendo por parte de los organismos regulatorios de dicho país armonizar la información de los prospectos ${ }^{(50)}$.

En Uruguay, las presentaciones comerciales disponibles se han registrado en miligramos para la administración parenteral y en mg y millones de unidades internacionales para nebulizar, estando declaradas como colistina base activa y como CMS.

El principal inconveniente es la forma en que la industria farmacéutica expresa el contenido de colistina en el rotulado del envase primario y secundario, así como en el inserto, lo que induce a errores al momento de proceder con la indicación, ya que no se especifica claramente si el vial contiene miligramos de colistina (como CBA) o de CMS.

En la tabla 2 se presenta la equivalencia entre colistina base activa y el CMS, que es la referencia a utilizar para los cálculos de la dosificación de colistina ${ }^{(13,26)}$.

\section{Metodología}

Con el objetivo de mejorar la comprensión referente a la rotulación de la dosis a administrar de colistina se revisaron los insertos y envases primarios de las presenta- ciones de colistina comercialmente disponibles en Uruguay, y se enviaron comunicaciones formales a las cinco direcciones de los laboratorios responsables de su registro, a efectos de su expedición formal en cuanto a la actividad de colistina base de sus formulaciones.

\section{Resultados}

Al momento de escribir esta revisión, en ninguno de los insertos de las diferentes presentaciones comercializadas en nuestro país se especifica a qué corresponden los miligramos contenidos en el vial. Habiendo consultado a cada uno de los proveedores, los mismos informaron que se refiere a la actividad de colistina base, es decir sobre la que de hecho hay que realizar el cálculo, partiendo de una dosis recomendada de 2,5 a $5 \mathrm{mg} / \mathrm{kg} / \mathrm{día}$ de actividad de colistina base y ajustando esta dosis de acuerdo a la función renal. Se presenta la información recabada en la tabla 3 .

Las formulaciones para nebulizar se presentan en mg y en mUI; en este escenario, se debe aplicar la conversión a partir de las equivalencias descritas en la tabla 2 de este documento.

Ejemplos prácticos: acordes a las recomendaciones de dosis de la FDA de colistina en base a miligramos por peso ideal.

\section{Caso 1}

$\mathrm{Si}$ en el centro hospitalario la presentación disponible es Avagal ${ }^{\circledR} 150 \mathrm{mg}$ para uso inyectable, y el paciente es una mujer para cuya talla el peso ideal es de $60 \mathrm{~kg}$, corresponde administrar una dosis carga de $5 \mathrm{mg} / \mathrm{kg} /$ día, es decir $300 \mathrm{mg}$, lo que equivale a dos frascos ampollas (viales) y luego de 12 horas una dosis de mantenimiento de 2,5 a $5 \mathrm{mg} / \mathrm{kg} /$ día dividida en dos dosis, es decir de 75 a $150 \mathrm{mg}$ cada 12 horas (un vial cada 12 horas) si presenta un clearance de creatinina mayor a $80 \mathrm{ml} / \mathrm{min}$.

\section{Caso 2}

Si la presentación disponible es Colistina Richet ${ }^{\circledR} 100$ mg en la misma situación, se deberían administrar tres viales como dosis carga y luego de 12 horas un frasco y medio cada 12 horas. El mismo principio aplica si se dispone de las presentaciones correspondientes a Colistina Permatec ${ }^{\circledR}$ o Alfacolin ${ }^{\circledR}$.

\section{Recomendaciones}

Los cálculos para prescripción según peso del paciente se deben realizar en miligramos de actividad de colistina base por peso ideal en función del sexo y de la talla.

A efectos de prevenir confusiones durante la prescripción médica, los responsables por su comercialización en Uruguay deberían especificar en forma explícita en prospectos, envases primario (etiqueta) y secundario 


\begin{tabular}{|c|c|c|c|c|c|c|}
\hline Nombre comercial & Laboratorio & $\begin{array}{l}\text { Composición según } \\
\text { inserto }\end{array}$ & $\begin{array}{c}\text { Actividad de } \\
\text { colistina base } \\
\text { (mg) }\end{array}$ & $\begin{array}{c}\text { Actividad de } \\
\text { colistina base (mUI) }\end{array}$ & $\begin{array}{l}\text { Colismetato sódico } \\
\text { (mg) }\end{array}$ & Administración \\
\hline \multirow[t]{2}{*}{ Avagal } & \multirow[t]{2}{*}{ Roemmers } & \multirow[t]{2}{*}{ Colismetato sódico } & 50 & ND & ND & NB \\
\hline & & & 150 & ND & ND & IM / IV \\
\hline Colistina Richet & Bioxel-Richet & $\begin{array}{l}\text { Colistina metasulfonato } \\
\text { de sodio }\end{array}$ & 100 & 3 & 240 & IM / IV / NB \\
\hline Alfacolin & Cipharma-Catalysis & $\begin{array}{l}\text { Colistina metasulfonato } \\
\text { de sodio }\end{array}$ & 100 & ND & ND & IM / IV / NB \\
\hline \multirow[t]{2}{*}{ Toliscrin } & \multirow[t]{2}{*}{ Lidertis-Dosa } & \multirow[t]{2}{*}{ Colistimetato sódico } & ND & 1 & ND & $\mathrm{INH}$ \\
\hline & & & ND & 2 & ND & INH \\
\hline Colistina Permatec & Rixdal-Permatec & $\begin{array}{l}\text { Colistina metasulfonato } \\
\text { de sodio }\end{array}$ & 100 & ND & ND & IM / IV / NB \\
\hline
\end{tabular}

(estuche), el contenido de su presentación comercial en cuanto a:

- colistimetato sódico en mg;

- colistina como actividad de colistina base (sustancia activa) en mg;

- colistina como actividad de colistina base en UI (si corresponde).

La información que presenta el producto debería ser claramente comprensible y no generar dudas respecto del contenido de los viales. Si el vial y el prospecto mencionan equivalencias, deberían coincidir con las equivalencias de la tabla 1 , siendo el factor de conversión entre CMS y colistina base de 2,4 (la potencia de 2,4 $\mathrm{mg}$ de $\mathrm{CMS}=$ potencia de $1 \mathrm{mg}$ de CBA).

\section{Conclusiones}

En esta era posantibiótica, la emergencia de resistencia obliga al clínico al empleo de moléculas en desuso en espera del surgimiento de nuevos fármacos. La optimización del uso de estos agentes en función de los datos de PK/PD/PG (farmacogenéticos) tiene un impacto directo en la morbimortalidad de los pacientes. La colistina es parte fundamental de los planes terapéuticos contra bacilos gramnegativos resistentes siendo necesario actualizar la información disponible para su uso seguro, evitando la emergencia de resistencia. Forma parte de esta estrategia una clarificación en los productos comercializados para no incurrir en errores en la prescripción, enmarcados en lineamientos de prácticas seguras.

\section{Agradecimientos}

A todos aquellos colegas que se plantean preguntas al momento de prescribir un fármaco, en particular a aquellos en los que el conocimiento sobre la forma óptima de administración es aún escaso.

Al Dr. Jordan Tenzi, que nos ha aportado la evidencia actual sobre la dosificación de colistina en la disfunción renal y en el paciente sometido a hemodiálisis.

\section{Abstract}

In the recent 10 years, in this post-antibiotic era, colisitin has reappeared as the last resource to face infections caused by Pseudomonas aeruginosa, Acinetobacter baumannii complex and carbapenemase-producing enterobacteriaceae. At some point the use of colistin was discontinued given its potentially severe side effects, such as nephro and neurotoxicity, although it reemerges today as an essential part of antibiotic plans when facing extremely resistant pathogens. This increase in the use of colistin has led to an antibiotic resistance emergency and thus today we face a potentially catastrophic situation. This happens in particular in connection with the recently described presence of transferable plasmids among species containing genes that confer resistance to colistin (gen mcr-1), a mechanism also identified in our country.

This review describes in detail the right dosing with the need to make load doses to achieve the appropriate therapeutic levels, based on current knowledge on pharmacokinetics and pharmacodynamics, as well as practi- 
cal aspects in the administration of the drug in cases of postsurgical meningitis/ventriculitis and the use by nebulization for the treatment of ventilation associated pneumonia. The study points out the need to use colistin along with another in-vitro active antibiotic, especially in critical patients and those with a creatinine clearance over $80 \mathrm{ml} / \mathrm{min}$. Dual pharmacotherapy is necessary in particular if the pathogen's minimum inhibitory concentration (MIC) is higher than $1 \mathrm{mg} / \mathrm{min}$, due to intra-treatment risk of sub-dosing and resistance emergency.

Likewise, in second section, the study addresses the complex nature of dosing given by the different presentations available in the market at the national level, what has resulted in dosage errors, and thus a higher risk of toxicity. The insets and primary packaging of colistin presentation available in the Uruguayan marketing were reviewed with the purpose of improving understanding in connection with the labeling of the doses to be administered. With that information, the pharmaceutical industries agents will be formally asked in writing to inform the colistin content (active drug) and its prodrug colistimethate sodium. Last, a number of recommendations were prepared as to the Information the authors understand should appear in the different presentations of colistin that is available in the market, to avoid prescription errors.

\section{Resumo}

Nos últimos 10 anos, nesta era pós-antibiótica, a colistina reapareceu como um último recurso para enfrentar infecções por patógenos como Pseudomonas aeruginosa, Acinetobacter baumannii complexo e enterobactérias produtoras de carbapenemases. Durante um período não foi utilizada devido a seus efeitos adversos potencialmente graves como a nefro e a neurotoxicidade, porém atualmente é parte fundamental da antibioticoterapia nos casos de patógenos extremadamente resistentes. O aumento de seu uso levou ao aparecimento de resistência e como consequência, de uma situação potencialmente catastrófica, especialmente a recentemente descrita presença de plasmídeos transferíveis entre espécies que contém genes que conferem resistência a colistina (gen mcr-1), mecanismo detectado também no Uruguai.

Nesta revisão, baseados nos conhecimentos atuais sobre a farmacocinética e farmacodinâmica, descreve-se detalhadamente a dosagem apropriada com necessidade de realizar doses carga para alcançar os niveles terapêuticos adequados, e os aspectos práticos da administração nos casos de meningite/ventriculite pós-operatória e de seu emprego na nebulização para o tratamento da pneumonia associada à ventilação. Destaca-se a necessidade de seu uso combinado com outro antibiótico ativo in vitro, especialmente em pacientes críticos e naqueles com clearance de creatininemia maior a 80 $\mathrm{ml} / \mathrm{min}$. A bi terapia é necessária principalmente se a concentração inibitória mínima do patógeno é maior a 1 $\mathrm{mg} / \mathrm{l}$, devido ao risco de subnotificação e o aparecimento de resistência intratratamiento.

Na segunda parte, discute-se a complexidade da dosagem em função das diferentes apresentações comercializadas no país que levaram a erros na posologia, com um maior risco de toxicidade. Buscando melhorar a compreensão dos aspectos relacionados a rotulação da dose de colistina revisaram-se as bulas e as embalagens primárias das apresentações de colistina comercialmente disponíveis no Uruguai, para solicitar formal e documentalmente às empresas farmacêuticas representantes, informação sobre a dosagem de colistina (droga ativa) e de seu precursor o colistimetato de sódio (CMS). Finalmente elabora-se uma série de recomendações com relação à informação que os autores consideram que deveria ser oferecida nas diferentes apresentações de colistina comercialmente disponíveis para não induzir a erros na prescrição.

\section{Bibliografía}

1. Rodríguez-Baño J, Cisneros JM, Cobos-Trigueros N, Fresco G, Navarro-San Francisco C, Gudiol C, et al; Study Group of Nosocomial Infections (GEIH) of the Spanish Society of Infectious Diseases, Infectious Diseases (SEIMC). Diagnosis and antimicrobial treatment of invasive infections due to multidrug-resistant Enterobacteriaceae: guidelines of the Spanish Society of Infectious Diseases and Clinical Microbiology. Enferm Infecc Microbiol Clin 2015; 33(5):337.e1-337.e21.

2. Paciel D, Palacio R, Bálsamo A, Moreira M, Hernández N, Cabeza E. Detección precoz y control de un brote de Pseudomonas aeruginosa productora de metalo-betalactamasa. Poster presentado en $43^{\circ}$ Congreso Nacional de Medicina Interna, IV Congreso Nacional de Infectología. 2 al 4 de Noviembre 2016. Montevideo, Uruguay.

3. Buroni M, Medina Presentado JC, Rieppi G, Paciel D. Consenso: uso de antimicrobianos en infecciones por microorganismos multi y panresistentes y guías para el tratamiento de bacterias productoras de KPC, agosto 2011. Montevideo: Cátedra de Enfermedades Infecciosas UDELAR, 2011. Disponible en: http://www.infectologia.edu.uy/publicaciones/uso-de-antimicrobianos-en-infecciones-por-microorganismos-multi-y-panresistentes-y-guias-para-el-tratamiento-de-bacterias-productoras-de-kpc-agosto-2011. [Consulta: 20 marzo 2017].

4. Levy Hara G, Gould I, Endimiani A, Pardo PR, Daikos G, Hsueh PR, et al. Detection, treatment, and prevention of carbapenemase-producing Enterobacteriaceae: recommendations from an International Working Group. J Chemother 2013; 25(3):129-40. 
5. Rigatto MH, Oliveira MS, Perdigão-Neto LV, Levin AS, Carrilho CM, Tanita MT, et al. Multicenter prospective cohort study of renal failure in patients treated with colistin versus polymyxin B. Antimicrob Agents Chemother 2016; 60(4):2443-9.

6. Vardakas KZ, Falagas ME. Colistin versus polymyxin B for the treatment of patients with multidrug-resistant Gram-negative infections: a systematic review and meta-analysis. Int J Antimicrob Agents 2017; 49(2):233-238.

7. Nation RL, Li J, Cars O, Couet W, Dudley MN, Kaye KS, et al. Consistent global approach on reporting of colistin doses to promote safe and effective use. Clin Infect Dis 2014; 58(1):139-41.

8. Nation RL, Velkov T, Li J. Colistin and polymyxin B: peas in a pod, or chalk and cheese? Clin Infect Dis 2014; 59(1):88-94.

9. Liu YY, Wang Y, Walsh TR, Yi LX, Zhang R, Spencer J, et al. Emergence of plasmid-mediated colistin resistance mechanism MCR-1 in animals and human beings in China: a microbiological and molecular biological study. Lancet Infect Dis 2016; 16(2):161-8.

10. Uruguay. Universidad de la República. Facultad de Medicina. Departamento de Bacteriología y Microbiología. Dos aislamientos clínicos de $E$. coli portadores de mcr-1 provenientes de dos centros de Montevideo. Montevideo: Departamento de Bacteriología y Microbiología, 2016.

11. Puig JM. Colistin: errores terapéuticos frecuentes con su utilización. Buenos Aires: Universidad Abierta Interamericana, 2015. Disponible en: https://sites.google.com/site/farmacologiamedicinauai/uso-racional-de-medicamentos/colistin-errores-terapeuticos. [Consulta: 20 enero 2017].

12. Gauthier TP, Lantz E, Frederick C, Masmouei H, Ruiz-Serrano L, Smith L, et al. Variability within investigations of intravenous colistin: the scope of the problem. Clin Infect Dis 2014; 58(9):1340-2.

13. Vidal Group Drug Information Systems. Colistiméthate sodique: mise à jour 24 Avril 2014. Issy-les-Moulineaux, Francia: Vidal Group, 2014. Disponible en: https://www.vidal.fr/substances/3936/colistimethate_sodique/. [Consulta: 20 enero 2017].

14. Landersdorfer CB, Nation RL. Colistin: how should it be dosed for the critically ill? Semin Respir Crit Care Med 2015; 36(1):126-35.

15. MacLaren G, Spelman D. Colistin: an overview. En: UpToDate, Post TW (Ed), UpToDate, Waltham, MA last updated Aug 15, 2016. Disponible en: http://www.uptodate.com/contents/colistin-an-overview. [Consulta: 20 enero 2017].

16. European Committee on Antimicrobial Susceptibility Testing. Recommendations for MIC determination of colistin (polymyxin E) as recommended by the joint CLSI-EUCAST Polymyxin Breakpoints Working Group. Växjö, Sweden: EUCAST, 2016. Disponible en: http://www.eucast.org/fileadmin/src/media/PDFs/ EUCAST_files/General_documents/Recommenda-
tions_for_MIC_determination_of_colistin

March_2016.pdf. [Consulta 15 abril 2017].

17. European Committee on Antimicrobial Susceptibility Testing. Breakpoint tables for interpretation of MICs and zone diameters Version 7.1, valid from 2017-03-10. Växjö, Sweden: EUCAST, 2017. Disponible en: http://www.eucast.org/clinical_breakpoints/. [Consulta: 15 abril 2017].

18. Clinical and Laboratory Standards Institute. Performance standards for antimicrobial susceptibility testing. CLSI Supplement M100. 27 ed. Wayne, PA: CLSI, 2017.

19. Gibson GA, Bauer SR, Neuner EA, Bass SN, Lam SW. Influence of colistin dose on global cure in patients with bacteremia due to carbapenem-resistant gram-negative bacilli. Antimicrob Agents Chemother 2015; 60(1):431-6.

20. Cheah SE, Li J, Tsuji BT, Forrest A, Bulitta JB, Nation RL. Colistin and polymyxin B dosage regimens against acinetobacter baumannii: differences in activity and the emergence of resistance. Antimicrob Agents Chemother 2016; 60(7):3921-33.

21. Organización Panamericana de la Salud. Alerta epidemiológica: enterobacterias con resistencia tranferible a colistina, implicaciones para la salud pública en las Américas 10 de junio 2016. Washindton, DC: OPS, 2016. Disponible en: http://antimicrobianos.com.ar/ATB/wp-content/uploads/2016/06/Enterobacterias-con-resistencia-transferible-a-colistina.pdf. [Consulta: 15 abril 2017].

22. Aguayo A, Mella S, Riedel G, Bello H, Domínguez M, González-Rocha G. Colistín en la era post-antibiótica. Rev Chil Infectol 2016; 33(2):166-76.

23. Martínez-Martínez L. Muerte bacteriana y heterorresistencia a los antimicrobianos. Enferm Infecc Microbiol Clin 2008; 26(8):481-4.

24. Li J, Rayner CR, Nation RL, Owen RJ, Spelman D, Tan $\mathbf{K E}$, et al. Heteroresistance to colistin in multidrug-resistant Acinetobacter baumannii. Antimicrob Agents Chemother 2006; 50(9):2946-50.

25. Nation RL, Garonzik SM, Li J, Thamlikitkul V, Giamarellos-Bourboulis EJ, Paterson DL, et al. Updated US and european dose recommendations for intravenous colistin: how do they perform? Clin Infect Dis 2016; 62(5):552-558.

26. Markantonis SL, Markou N, Fousteri M, Sakellaridis N, Karatzas S, Alamanos I, et al. Penetration of colistin into cerebrospinal fluid. Antimicrob Agents Chemother 2009; 53(11):4907-10.

27. Jiménez-Mejías ME, Pichardo-Guerrero C, Márquez-Rivas FJ, Martín-Lozano D, Prados T, Pachón J. Cerebrospinal fluid penetration and pharmacokinetic/pharmacodynamic parameters of intravenously administered colistin in a case of multidrug-resistant Acinetobacter baumannii meningitis. Eur J Clin Microbiol Infect Dis 2002; 21(3):212-4.

28. Karaiskos I, Galani L, Baziaka F, Giamarellou H. Intraventricular and intrathecal colistin as the last therapeutic resort for the treatment of multidrug-resistant and extensively drug-resistant Acinetobacter baumannii ventriculitis and me- 
ningitis: a literature review. Int J Antimicrob Agents 2013; 41(6):499-508.

29. Fried HI, Nathan BR, Rowe AS, Zabramski JM, Andaluz $\mathbf{N}$, Bhimraj A, et al. The insertion and management of external ventricular drains: an evidence-based consensus statement. A statement for healthcare professionals from the Neurocritical Care Society. Neurocrit Care 2016; 24(1):61-81.

30. Fotakopoulos G, Makris D, Chatzi M, Tsimitrea E, Zakynthinos E, Fountas K. Outcomes in meningitis/ventriculitis treated with intravenous or intraventricular plus intravenous colistin. Acta Neurochir (Wien) 2016; 158(3):603-10.

31. Garonzik SM, Li J, Thamlikitkul V, Paterson DL, Shoham S, Jacob J, et al. Population pharmacokinetics of colistin methanesulfonate and formed colistin in critically ill patients from a multicenter study provide dosing suggestions for various categories of patients. Antimicrob Agents Chemother 2011; 55(7):3284-94.

32. Dudhani RV, Turnidge JD, Coulthard K, Milne RW, Rayner CR, Li J, et al. Elucidation of the pharmacokinetic/pharmacodynamic determinant of colistin activity against Pseudomonas aeruginosa in murine thigh and lung infection models. Antimicrob Agents Chemother 2010; 54(3): 1117-24.

33. Dudhani RV, Turnidge JD, Nation RL, Li J. fAUC/MIC is the most predictive pharmacokinetic/pharmacodynamic index of colistin against acinetobacter baumannii in murine thigh and lung infection models. J Antimicrob Chemother 2010; 65(9):1984-90.

34. Falagas ME, Kasiakou SK. Toxicity of polymyxins: a systematic review of the evidence from old and recent studies. Crit Care 2006; 10(1):R27.

35. De Bonis P, Lofrese G, Scoppettuolo G, Spanu T, Cultrera $\mathbf{R}$, Labonia $\mathbf{M}$, et al. Intraventricular versus intravenous colistin for the treatment of extensively drug resistant Acinetobacter baumannii meningitis. Eur J Neurol 2016; 23(1):68-75.

36. Johns Hopkins Medicine. Antibiotic guidelines 2015-2016: treatment recommendations for adult inpatients. Baltimore, Maryland: Johns Hopkins Medicine, 2016. Disponible en: www.hopkinsmedicine.org/amp/guidelines/antibiotic_guidelines.pdf. [Consulta: 15 abril 2017].

37. Bihan K, Lu Q, Enjalbert M, Apparuit M, Langeron O, Rouby JJ, et al. Determination of colistin and colistimethate levels in human plasma and urine by high-performance liquid chromatography-tandem mass spectrometry. Ther Drug Monit 2016; 38(6):796-803.

38. Mercier T, Tissot F, Gardiol C, Corti N, Wehrli S, Guidi M, et al. High-throughput hydrophilic interaction chromatography coupled to tandem mass spectrometry for the optimized quantification of the anti-Gram-negatives antibiotic colistin $\mathrm{A} / \mathrm{B}$ and its pro-drug colistimethate. J Chromatogr A 2014; 1369:52-63.
39. Nation RL, Garonzik SM, Thamlikitkul V, Giamarellos-Bourboulis EJ, Forrest A, Paterson DL, et al. Dosing guidance for intravenous colistin in critically-ill patients. Clin Infect Dis 2017; 64(5):565-571.

40. Dalfino L, Puntillo F, Mosca A, Monno R, Spada ML, Coppolecchia S, et al. High-dose, extended-interval colistin administration in critically ill patients: is this the right dosing strategy?: a preliminary study. Clin Infect Dis 2012; 54(12):1720-6.

41. Tunkel AR, Hasbun R, Bhimraj A, Byers K, Kaplan SL, Michael Scheld W, et al. 2017 Infectious Diseases Society of America's Clinical Practice Guidelines for Healthcare-Associated Ventriculitis and Meningitis. Clin Infect Dis 2017 Feb 14. [Epub ahead of print].

42. Grille P, Bertullo M. Infecciones posneuroquirúrgicas. En: Biestro A. CTI protocolos. Montevideo: Editorial Cuadrado, 2015: 43-8.

43. Kalil AC, Metersky ML, Klompas M, Muscedere J, Sweeney DA, Palmer LB, et al. Management of adults with hospital-acquired and ventilator-associated pneumonia: 2016 clinical practice guidelines by the Infectious Diseases Society of America and the American Thoracic Society. Clin Infect Dis 2016; 63(5):e61-e111.

44. Wenzler E, Fraidenburg DR, Scardina T, Danziger LH. Inhaled antibiotics for gram-negative respiratory infections. Clin Microbiol Rev 2016; 29(3):581-632.

45. Ari A. Jet, ultrasonic, and mesh nebulizers: an evaluation of nebulizers for better clinical outcomes. Eurasian J Pulmonol 2014; 16(1):1-7

46. Bassetti M, Luyt CE, Nicolau DP, Pugin J. Characteristics of an ideal nebulized antibiotic for the treatment of pneumonia in the intubated patient. Ann Intensive Care 2016; 6(1):35.

47. Vincent JL, Bassetti M, François B, Karam G, Chastre J, Torres A, et al. Advances in antibiotic therapy in the critically ill. Crit Care 2016; 20(1):133.

48. Dhanani J, Fraser JF, Chan HK, Rello J, Cohen J, Roberts JA. Fundamentals of aerosol therapy in critical care. Crit Care 2016; 20(1):269.

49. Rello J, Solé-Lleonart C, Rouby JJ, Chastre J, Blot S, Poulakou G, et al. Use of nebulized antimicrobials for the treatment of respiratory infections in invasively mechanically ventilated adults: a position paper from the European Society of Clinical Microbiology and Infectious Diseases. Clin Microbiol Infect; 2017. pii: S1198-743X(17)30219-7. doi: 10.1016/j.cmi.2017.04.011.

50. Sociedad Argentina de Infectología. Actualización: alerta presentaciones comerciales colistina, disponibilidad de polimixina B en Argentina. Buenos Aires: SADI, 2015. Disponible en: http://antimicrobianos.com.ar/ATB/wp-content/ uploads/2015/09/Actualizaci\%C3\%B3n-alerta-colistina-julio-2015-2.pdf. [Consulta: 15 abril 2017]. 